Recent Insights into the Physics of the Sun and Heliosphere:

Highlights from $\mathrm{SOHO}$ and Other Space Missions

IAU Symposium, Vol. 203, 2001

P. Brekke, B. Fleck, and J. B. Gurman eds.

\title{
Magnetic Configuration and Evolution in a Solar Active Region
}

\author{
Y. Liu and H. Zhang \\ Beijing Astronomical Observatory, NAOCAS, A20 Datun Road, \\ Chaoyang District, Beijing, 100012 China
}

\begin{abstract}
We present results of the analysis of NOAA 8668, which was observed successively by space satellite $(\mathrm{SOHO})$ and ground-based observatories (BBSO, Huairou). The combined observation offers us a good example of a region observed from low to high solar atmosphere. Several flares and a sigmoid filament were observed in the AR, and we observed the sigmoid filament from its birth to disintegration. The configuration of the magnetic field of the AR changed quickly as well as the loops. From EIT movies, we can even judge the sign of the sigmoid filament's magnetic helicity. The forming and heating of the loops were the result of magnetic reconnection, and the corona seemed heated when the loops became opened.
\end{abstract}

\section{Introduction}

The heating of the corona is one puzzle in solar physics. The corona sustain the solar wind with energy, and it plays an important role in the formation of CME, which may yield unforeseen consequences to Sun-Earth environment. Coronal loops are treated as traces of magnetic force lines rooting at solar surface, understanding the interdependence between the magnetic field and evolution of loops will contribute to solve the stubborn problem of coronal heating. From satellite data (TRACE, SOHO, YOHKOH), we can see that there is an obvious simple correlation between strong magnetic fields and bright coronal features. Magnetic loops oscillations following an eruption, such as a flare, was a new discovery of the activation of magnetic field on coronal loops (Aschwanden et al 1999). Meanwhile, filament is another important phenomena, whose formation, activation and eruption or disappearance are associated closely with the evolution of magnetic field. In the paper we attempt to make it clear the correlation between magnetic field's evolution and coronal getting heated.

\section{Observation and results}

The data used in the work, are EUV images $(195 \AA, 284 \AA)$ taken by $S O H O / E I T$, full-disk $S O H O / \mathrm{MDI}, \mathrm{H} \alpha$ images and magnetic field data taken by BBSO and Huairou. These data are about from Aug 14 to Aug 26 1999. We have translated them into movies, from which we can see that the AR (NOAA 8668) evoluted quickly during Aug 14-20, especially during on the midian disk(Aug 18-20). 


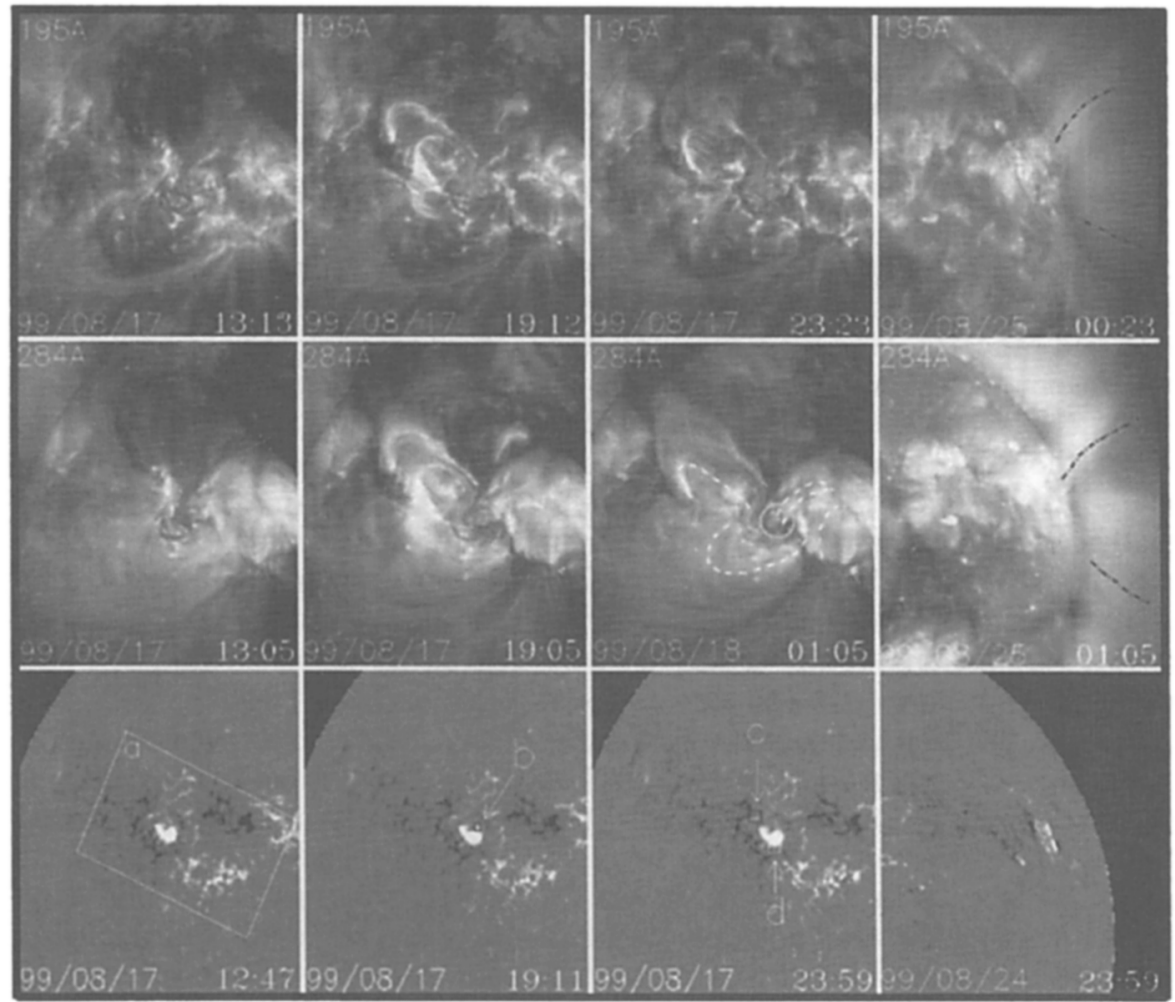

Figure 1. Configuration and evolution of the AR(see text).

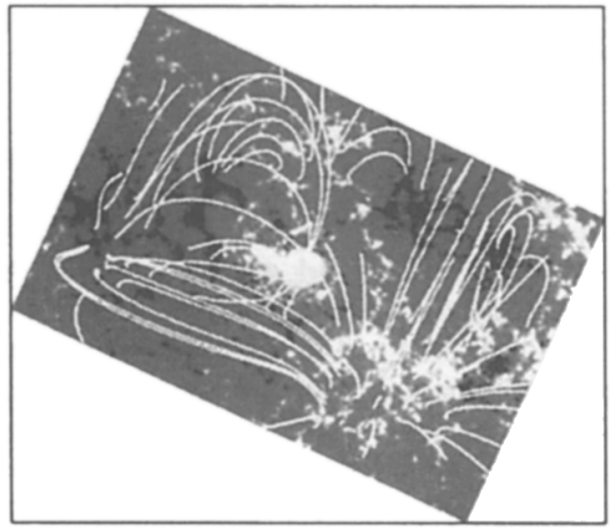

Figure 2. Extrapolated potential field based on Huairou magnetic field. 
Magnetic flux emergences and cancellations took place strenuously between some regions of the main positive polarity with their enhanced negative networks respectively. The sigmoid filament was formed during Aug 15-17 in an inverse S shape, full developed on Aug 18, and then began to break late on Aug 19. From Aug 20, the filament disintegrated and disappeared obviously in $\mathrm{H} \alpha$ images.

The magnetic fields around the filament were very complicated (fig2). Especially the quick changes in its local regions(such as $b, c, d$ in fig1). The region c was where the filament broke on late Aug 19, opposite elements continuously collided and canceled. The filament's tail was located through the region d south of the sunspot, where many point-like positive magnetic elements emerged and moved quickly. From Doppler images of Aug 20, we found the tail moved upward and downside in a vibratile way, and bifurcated into several small long tiny silks associated with at least 3 flares. After which, the total filament disappeared gradually. The loops' evolution mimiced that of the filaments shown in EUV movies. At about 13:05 UT Aug 17, the loops began to emerge and rise after a flare, when the filament came into being. The loops reached their peak at about 19:05 UT, and got stabled since 01:05 UT, Aug 18, when the filament had matured. The scenario were the same in both $195 \AA$ and $284 \AA$ (fig1).

Based on the ground magnetic field data of Huairou, we extrapolated region a by Green function method(Chiu Y. 1977) and compared it with EIT images. It's shown that these coronal loops chiefly consisted of three magnetic systems, but one of them obviously deviated from the EUV images which is shown in (01:05 UT of Aug 18 in fig1, fig2). In the first image, the north-east loop connected the negative network with positive network of the sunspots, but not with the sunspots' field region as in fig2, which was indicated by a gray circle. While the northeast loop of the last image connected them two, so we can say that there's much non-potential energy reserved in the magnetic field morphology of north-east AR at that time, the energy must contribute to coronal heating. When the AR was at the west disk edge, the morphology of the loops became opened, and the filament had disappeared. From EUV images, we can see that the corona was brighter in $284 \AA$ than in $195 \AA$ indicating it had been heated.

There's an interesting phenomenon that we see in the movies, i.e., the filament chirality may be determined by the case of how bright and dark threads overlapped each other (Chae J. 2000).

\section{Conclusion and discussion}

We have examined the configuration and evolution of NOAA 8668 from the angles of a sigmoid filament and the EUV loops and coronal heating. The loops' evolution mimics that of the filament. Deep carefully calculations will be done in the future work to research the heating mechanism of the corona.

\section{References}

Aschwanden et al., 1999, ApJ, 520, 880

Chae Jongchui, 2000, ApJ, 540, L115

Chiu Y. T. \& Hilton H. 1977, ApJ, 212, 873 